

\title{
Diagnóstico FACIL Empresarial, Finanzas, Auditoría, Contabilidad, Impuestos, Legal
}

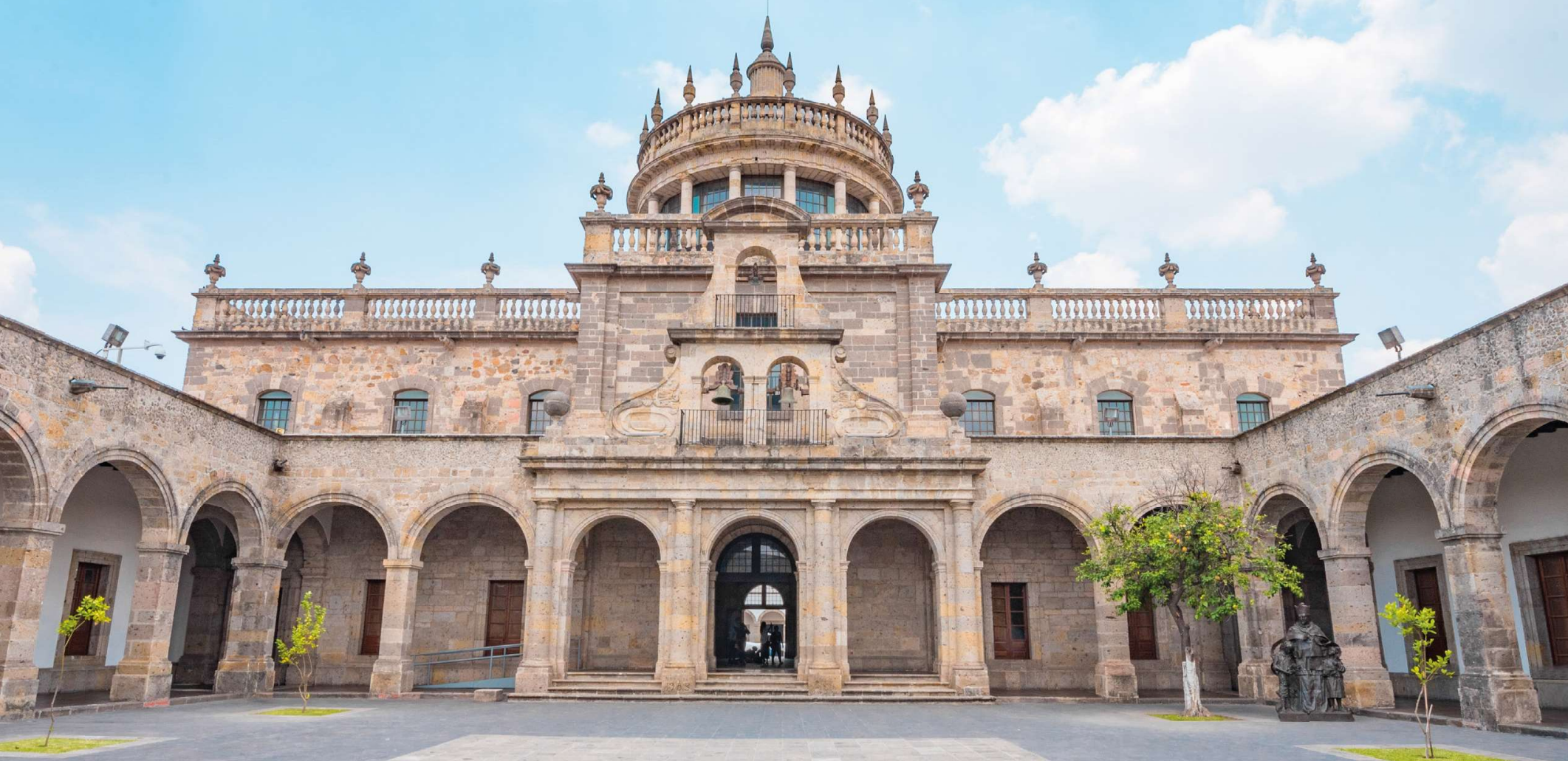




\section{Directorio}

\section{Consejo editorial}

Director de la Revista

Dr. Cristian Omar Alcantar López

Universidad de Guadalajara

Editor Responsable

Dr. Marco Antonio Daza Mercado

Universidad de Guadalajara

Revisión y composición

Miguel Ángel Serrano Núñez

Diseño de portada

Lic. Daniel García Arellano

Soporte técnico

Lic. Sergio Ricardo Palomares Arias

\section{Consejo Editorial Interno}

Dr. Francisco de Jesús Mata Gómez

Universidad de Guadalajara

Dr. Javier Ramírez Chávez

Universidad de Guadalajara

Dr. Alejandro Campos Sánchez

Universidad de Guadalajara

Dr. Sergio Sánchez Enríquez

Universidad de Guadalajara

Dr. José Trinidad Ponce Godínez

Universidad de Guadalajara

\section{Consejo Editorial Externo \\ Internacionales}

Dr. Ricardo Pahlen Acuña

Universidad de Buenos Aires (Argentina)

Dr. Francisco Borrás Atiénzar

Universidad de La Habana (Cuba)

Dra. Begoña Prieto Moreno

Universidad de Burgos (España)

Dra. Ana de Dios Martínez

Universidad de Camagüey (Cuba)

Dra. Leticia Ortiz Torricos

Universidad Autónoma Gabriel René Moreno (Bolivia)

Dra. Cecilia Rita Ficco

Universidad Nacional de Río Cuarto (Argentina)

Dr. Ricardo Alonso Colmenares Flórez

Corporación Universitaria U de Colombia (Colombia)

Dr. Gonzalo Wandosell Fernández de Bobadilla

Universidad Católica de Murcia (España)

\section{Nacionales}

Dr. Jesús María Martín Terán Gastelúm

Universidad Estatal de Sonora (México)

Dr. José Manuel Osorio Atondo

Universidad Estatal de Sonora (México)

Dr. Oscar González Muñoz

Universidad Veracruzana (México)

Dr. Juan Manuel Ortega Maldonado

Universidad Autónoma de Morelos (México)

Dra. Leticia María González Velasquez

Universidad de Sonora (México)

Dr. Oscar Bernardo Reyes Real

Universidad de Colima (México)

Dr. Jerónimo Ricárdez Jiménez

Universidad Veracruzana (México)

Dra. Aurea Arellano Cruz

Universidad de la Sierra Sur (México) 


\title{
PIAGNÓSTICO
IFACIL
IEMPRESARIAL
}

\section{Efectos de la modernización en la recaudación de la aduana de Lázaro Cárdenas}

\author{
Óscar Bernardo Reyes Real / Universidad de Colima / oscarreal@ucol.mx \\ Ariel Gutiérrez Ortiz / Universidad de Colima / agutierrez18@ucol.mx \\ Ana María del Rosario Álvaro Obregón / Universidad de Colima / oregon.ana@gmail.com
}

Fecha de recepción: 28/12/2020

Fecha de Aceptación: 05/02/2021
Fecha de revisión: 14/01/2021

Fecha de publicación: 10/03/2021

\section{Resumen}

El objetivo de esta investigación fue determinar las tendencias de la modernización en la Aduana de Lázaro Cárdenas y sus efectos en la función de recaudación, esto a través de la propuesta de un modelo para el estudio de las mejores prácticas de la modernización de la Aduana de Lázaro Cárdenas y su efecto en las funciones sustantivas de su gestión, específicamente a partir de la réplica de un modelo medible de modernización aduanera con variables observadas a través de encuestas. La metodología empleada fue el análisis factorial confirmatorio que utiliza los modelos de ecuaciones estructurales o $\mathrm{SEM}^{1}$ por sus siglas en inglés.

Los resultados mostrados a partir de la metodología empleada muestran que todas las reformas legales y programas del gobierno federal de México para la administración pública aduanera tendientes a su modernización han influido positivamente en la recaudación de la aduana de Lázaro Cárdenas.

Advirtiéndose que las mejoras en la recaudación de la aduana de Lázaro Cárdenas provienen con mayor intensidad de las mejoras que se han efectuado en materia de infraestructura y de los cambios tecnológicos tales como inserción de la ventanilla digital, sistema electrónico aduanera, módulo de administración tributaria del comercio exterior y proyecto de integración tecnológica aduanera.

Palabras clave: Modernización, aduana, gestión aduanera, recaudación, ecuaciones estructurales, análisis factorial.
The objective of this research was to determine the trends of modernization in the Lázaro Cárdenas Customs and its effects on the collection function, this through the proposal of a model for the study of the best practices of the modernization of the Customs of Lázaro Cárdenas and its effect on the substantive functions of his management, specifically from the replication of the measurable model of customs modernization with variables observed through surveys prepared and proposed by Reyes (2018). The methodology used was the confirmatory factor analysis that uses the Structural Equation Models or SEM for its acronym in English.

The results shown from the methodology used show that all the legal reforms and programs of the federal government of Mexico for the customs public administration aimed at its modernization have positively influenced the collection of the Lázaro Cárdenas customs.

Noting that the improvements in the collection of Lazaro Cardenas customs come with greater intensity from the improvements that have been made in terms of infrastructure and technological changes such as insertion of the digital window, electronic customs system, tax administration module of the foreign trade and customs technological integration project.

Key words: Modernization, customs, customs management, collection, structural equations, factor analysis.

$1 \quad$ Structural equations modeling. 


\section{Introducción}

Como resultado de la globalización los países alrededor del mundo se encuentran altamente vinculados, sobre todo en el rubro económico, reconociéndose al comercio exterior como una de las actividades más importantes, por lo que el papel de las autoridades a quienes les competen las funciones relacionadas con el control y vigilancia de las fronteras se vuelve fundamental, generando entonces la imperiosa necesidad de que la gestión en las aduana se efectué bajo los principios de eficiencia, facilitación y transparencia.

Por lo expresado en el párrafo anterior, los organismos internacionales relacionados con el comercio exterior y las aduanas, como lo son la Organización Mundial del Comercio (OMC) y la Organización Mundial de Aduanas (OMA), han emitido normatividad internacional como el acuerdo sobre facilitación del comercio mejor conocido como el acuerdo de Bali (Organización mundial de comercio, 2013), el convenio de Kyoto (Convenio de Kyoto, 1974) y el marco normativo para asegurar y facilitar el comercio mundial (marco SAFE) (Organización Mundial de Aduanas, 2012), acuerdos tendientes a poner en marcha actos de modernización en las administraciones aduaneras del mundo con el objeto de ver mejorías en las funciones que se derivan de su gestión (Reyes, 2018).

La modernización aduanera en México ha considerado diversas dimensiones que constituyen su administración, aspectos que se pueden resumir principalmente en dos: organizacional y operativa (Reyes, 2018), reconociéndose como metas principales la mejora en la infraestructura física y tecnológica, cuestiones de organización, administración y procesos, recursos humanos y la calidad de los servicios públicos que reciben los contribuyentes usuarios de las aduanas (Administración General de Aduanas, 2007; Reyes, 2006; Servicio de Administración Tributaria, 2006).

Debido a la importancia del comercio internacional para México, el gobierno federal de México ha efectuado diversas reformas legales, programas y proyectos específicos, para la modernización y eficiencia de las aduanas, a través de la mejora de su infraestructura, la incorporación de sistemas y tecnologías de punta y la reingeniería de sus procesos, en la búsqueda del incremento de la efectividad en sus funciones sustanciales, generándose la coyuntura para la realización de estudios que busquen corroborar si es que las modificaciones a las leyes y los cambios institucionales descritas con antelación, han generado cambios positivos en cada una de las funciones sustanciales que se desprenden de la gestión aduanal, particularmente, en la recaudación aduanera.
La mayor parte de los estudios orientados al análisis de la modernización aduanera son de tipo descriptivo, es por eso que el propósito de esta investigación es realizar un estudio mediante el uso del modelo de ecuaciones estructurales mediante la aplicación del análisis factorial confirmatorio para determinar las tendencias de la modernización en la Aduana de Lázaro Cárdenas y sus efectos en la función de recaudación. Para la recolección de los datos se utilizó como técnica de investigación la encuesta estructurada y como instrumento para la recolección de la información un cuestionario con 9 ítems mediante el uso de una escala tipo Likert, que recolectó la percepción de los usuarios de la Aduana de Lázaro Cárdenas, México, respecto del objeto de estudio.

\section{Planteamiento del problema}

Trejo (2008), Reyes (2006) y Horta (2000) afirman que la necesidad de modernizar la gestión pública (GP) de las aduanas ha sido provocada por el incremento de las operaciones transfronterizas alrededor del mundo, la celeridad con el que la tecnología y los medios digitales han avanzado y la exigencia por parte de todos los involucrados en el comercio internacional respecto de la disminución de los costos que se generan por el comercio transfronterizo, siendo necesaria de forma indispensable la intervención eficaz, transparente y competitiva de las autoridades aduaneras que facilite el flujo de mercancías a través de las fronteras y un efectivo control en las mismas, circunstancia que tendrá como manifestación el aumento en la cantidad de transacciones, de la recaudación, en la detección de ilícitos por las aduanas y la excelencia en los servicios que se prestan a los agentes de aduanas, importadores y exportadores.

Reyes, Palos y De Dios (2018) afirman que han sido muchas las formas en que se ha tratado el análisis de la Nueva Gestión Pública (NGP) como paradigma de modernización en el sector público que ha trascendido también al ámbito de la gestión aduanera; por lo que de acuerdo a los diferentes contextos y particularidades de la organización pública que se esté estudiando, se han incluido diversas manifestaciones de la NGP tales como los sistemas de evaluación del desempeño (SED) (Culebro, 2008; Bozzi, 2014), profesionalizado y cambiado el perfil de los servidores públicos (Morales, 2014; Ramírez, 2013), se ha introducido la gestión de la calidad (Moyada, 2011; Ruiz, 2012), la innovación (Ávila, 2014; Sánchez, 2011), la búsqueda de la eficiencia como muestra del buen uso de los 
recursos públicos (Doimeadiós y Rodríguez, 2016; Zamora y Navarro, 2014; Morales, 2014), la orientación a los resultados (García, 2007) y el enfoque en el ciudadano (Bozeman, 2015), por mencionar algunas. No obstante, no todo ha funcionado de forma adecuada, encontrándose inconsistencias y fallos en la inserción de los postulados perseguidos por la NGP para la modernización de la gestión aduanera, en ese contexto, es relevante conocer a detalle cada institución y el contexto en el que se desarrolla y con base a ello hacer frente a las demandas socio económicas subsistentes.

Para países como México es claro que se ha avanzado en materia de gestión aduanera, siendo esta más eficaz, transparente y competitiva, empero, los análisis respecto de su modernización han girado en torno a las reformas considerando las funciones aduaneras de manera individual con un enfoque representativo y aunque se han hecho indagaciones sobre las mejores prácticas, como son los trabajos de Trejo (2008), Reyes (2006) y Horta (2000), no ha habido un perspectiva holística para corroborar lo afirmado dentro de la teoría sobre el modelo de costumbres de clase mundial que proponen autores como Coyle, Cruthirds, Naranjo, y Nobel (2014), limitándose a estudios de naturaleza descriptiva sin una comprobación empírica.

El modelo de Coyle et al. (2014) concibe a la modernización en aduanas mediante un constructo que está compuesto por varias dimensiones que son sintetizadas con base a la investigación de Reyes (2018) y para efectos de esta investigación, en solo tres dimensiones: función pública, infraestructura y cambios tecnológicos, incluyendo para los propósitos de este documento, solo el análisis de su efecto en la recaudación aduanera reconocida por los autores de referencia como una de las funciones aduaneras sustanciales en su gestión.

Las modificaciones jurídicas, especialmente a la ley aduanera vigente en México, han propiciado el empleo de mecanismos y sistemas apoyados en cambios tecnológicos y en tecnología de punta, generando importantes manifestaciones en la recaudación aduanera. De ahí que hay que considerar que, como afirma Trejo (2008), México se distingue por tener una de las aduanas más modernas en el mundo debido a su reorganización, uso de la más avanzada tecnología, equipamiento e infraestructura moderna.

Si bien es cierto que México advierte de forma clara momentos de reorganización, cambios en equipamiento y múltiples mejoras en relación a mecanismos y sistemas informáticos, que son cada vez más modernos con la aplicación de las mejores tecnologías; no obstante, no se había podido determinar el progreso en materia de eficiencia aduanera en el contexto de México, iniciando con los cambios en las funciones de la gestión aduanera, no obstante partiendo de las proposiciones de Coyle et al. (2014) y Reyes (2018), se puede explorar en qué magnitud se produce un resultado de las mejores prácticas particularmente en recaudación aduanera como una de las funciones claves de la aduana moderna y reconocida en esta investigación, y en qué medida esta función responde a las reformas de la función pública aduanera, los cambios tecnológicos y a la infraestructura.

Como se ha afirmado, los acontecimientos más relevantes en el tema de modernización aduanera se observaron en la Aduana de Lázaro Cárdenas con la implementación del Plan de Modernización de las Aduanas 2007-2012 (PMA), que se origina con el propósito de impulsar la eficiencia y productividad de la cadena logística nacional e internacional que ejecuta por el puerto mencionado, provocando una avance en la capacidad de esta aduana y afianza al puerto como el segundo más importante del país, el $2^{\mathrm{do}}$ más importante en el manejo de contenedores (Administración portuaria integral de Lázaro Cárdenas, 2019) y el $14^{\circ}$ de Latinoamérica y el Caribe, según la Comisión Económica para América Latina y el Caribe (2019). Es importante destacar también que en esta aduana se han introducido sistemas y mecanismos tecnológicos de punta, lo que evidencia avances en términos tecnológicos, tan es así que la misma está incluida dentro del Programa de Integración Tecnológica Aduanera (PITA) (Servicio de Administración Tributaria, 2016d).

Aunque el SAT (Servicio de Administración Tributaria, 2015a, 2015b, 2016b, 2016a) estableció indicadores para valorar el desempeño de este plan, tales como: personal que se contrató y capacitó, mejora en las instalaciones, adquisición de equipo e incorporación de tecnología para la fiscalización y gestión del despacho de mercancías y reestructuración administrativa, cambios y modificaciones en el organigrama y estructura administrativa, incremento en la asertividad de detección de irregularidades, contabilizar sucesos que demuestren y den precedente a los avances del proyecto, información estadística, alertas, eventos, detecciones y embarques, por mencionar algunos; para este estudio lo más relevante comprobar el efecto de las reformas tendientes a modernizar la gestión aduanera con el objeto de verificar si estas juegan un importante en la recaudación aduanera (Reyes, 2018).

La exigencia de conocer el estatus quo en materia de prácticas aduaneras con el objeto de identificar áreas de oportunidad para ser atendidas y con esto provocar su 
renovación, ha sido documentado tal y como se puede advertir del estudio efectuado por parte de la OMC en el año 2002, respecto de los resultados de las prácticas aduaneras actuales, análisis mediante el cual conoció las potenciales barreras en el comercio global (World Trade Organization, 1998), por lo que tal y como lo afirma Reyes, (2018, pag.16) resulta necesario identificar el efecto de las mejores prácticas en las operaciones aduaneras de los países y, en este caso, buscar adaptar el constructo teórico para las operaciones aduanales de clase mundial examinado en el contexto internacional y comparado con las prácticas actuales empleadas por oficina de aduanas y protección fronteriza de los Estados Unidos de Norteamérica de acuerdo a la investigación de Coyle et al. (2014) y analizar su impacto en las funciones sustantivas que se deben ejecutar por la administración pública aduanera, de forma especial en la recaudación aduanera.

Debido a la importancia que tiene el comercio internacional para México y el rol primordial de la Aduana de Lázaro Cárdenas en las transacciones transfronterizas por la vía marítima y con el objeto de elaborar propuestas abonen al incremento de la eficiencia aduanera, resulta indispensable responder la siguiente pregunta de investigación, como problema de esta investigación: ¿Cuánto de las mejoras en la recaudación aduanera es atribuible a las reformas en la función pública, los cambios tecnológicos y de infraestructura en la Aduana de Lázaro Cadenas?

De la identificación del problema de investigación se desprende el objetivo general de la indagación: Determinar cuánto de las mejoras en la facilitación del comercio exterior es atribuible a las reformas en la función pública, los cambios tecnológicos y de infraestructura en la Aduana de Lázaro Cadenas.

Del objetivo general se desprenden los siguientes objetivos específicos:

1. Precisar en qué medida la función pública como dimensión de la modernización aduanera genera un efecto en la función sustantiva de recaudación en la Aduana de Lázaro Cárdenas.

2. Determinar en qué medida la infraestructura, como dimensión de la modernización aduanera, genera un efecto en la función sustantiva de recaudación en la Aduana de Lázaro Cárdenas.

3. Determinar en qué medida los cambios tecnológicos como dimensión de la modernización aduanera genera un efecto en la función sustantiva de recaudación en la Aduana de Lázaro Cárdenas.

\section{Marco teórico y revisión de literatura}

\section{La modernización del sector público en el contexto mexicano}

La modernización de la administración pública en México proviene de las mejores prácticas en el gobierno que fueron insertadas a partir del reconocimiento de la Nueva Gestión Pública ${ }^{2}$ (NGP) como punto de partida para su crecimiento, sumado a todo esto se encuentra el trabajo de muchos funcionarios públicos en diversos periodos y que formaron parte de los tres poderes federales reconocidos en México y en los distintos niveles gubernamentales, reconociéndose los cambios como lo expresa (Reyes, 2018, pag. 34) desde Instituciones de Educación Superior (IES) hasta organizaciones civiles a través de estudios y análisis particulares fueron estableciendo las pautas a seguir.

De acuerdo con Ponce de León (2011), la globalización y los incesantes cambios tecnológicos, particularmente en comunicación e informática, fueron catalizadores de la evolución del estado, advirtiendo una alta correspondencia en individuos, organizaciones civiles, dependencias y dependencias de gobiernos de todos los niveles, que género como resultado la identificación de buenas experiencias en el estado asociadas con la atención de las exigencias ciudadanas. Entonces la presencia de la tecnología y su uso en el actuar gubernamental, elevo su transparencia, evidenciando los actos de corrupción e impulsando el reconocimiento de conceptos tales como el gobierno electrónico o e-goverment.

\section{La modernización del sector publico aduanero}

La aduana como parte de la administración pública no ha estado exenta de los efectos de la incorporación de los elementos de la NGP en su gestión, los organismos internacionales especializados en la materia tales como la Organización Mundial del Comercio (OMC) y la Organización Mundial de Aduanas (OMA), reconocen en las funciones de la aduana a nivel global una parte transcendental en la cadena de suministros, esto por la relevancia de la gestión de la administración pública aduanera en la política exterior, el desarrollo económico, el flujo del comercio exterior y la seguridad fronteriza en las naciones (Reyes, 2018, p. 45)

La modernización de las aduanas se reconoce a partir del convenio de Kyoto revisado (CKR) y el marco de estándares para asegurar y facilitar el comercio (Mar-

2 Para Alcantar, Daza y Velásquez (2019), la modernización es un proceso fundamental que permite garantizar el buen funcionamiento de las instituciones. 
co SAFE) de la OMA, convenios de donde se desprende las mejores prácticas aduaneras (Shujie y Shilu, 2009), practicas definidas como: “...procedimientos simples y eficaces a fin de dar el máximo nivel de facilitación a las operaciones aduaneras de mercancías y de pasajeros, así como de diversos procedimientos especiales" (Trejo, 2007, p. 222) definidas también por la Organización mundial del comercio (s. f.) como "metodologías modernas para establecer un equilibrio entre las funciones aduaneras de control y recaudación de ingresos fiscales, y la de facilitación del comercio", finalmente Coyle et al. (2014); Gwardzińska (2012) y World Bank (2016) coinciden que las mejores prácticas en materia aduanera en síntesis se expresan a través de seis constructos teóricos que son: infraestructura, procedimientos, tecnología, orientación en el tiempo, costo y misión, que permiten la elaboración de un constructo particular teniendo en cuenta las costumbres de clase mundial para las aduanas, en donde se localizan las mejores prácticas, que debieran seguir las aduanas para alcanzar su modernización y generar con- secuencias en sus funciones sustanciales, teniendo como resultado a partir del trabajo de Reyes (2018) que las dimensiones de la modernización aduanera a considerar en esta investigación son: Infraestructura, cambios tecnológicos y función pública.

Ahora bien, tomando como punto de partida el constructo teórico de Coyle et al. (2014), "modelo teórico de costumbres de clase mundial"; se asumirán como componentes de la modernización aduanera las tres dimensiones sugeridas por Reyes (2018): infraestructura, cambios tecnológicos y función pública, elementos con un relevante impacto en las funciones esenciales reconocidas en la gestión de la administración pública aduanera de acuerdo a la síntesis teórico en Reyes (2018) que se muestra en la tabla 1, con base en Barahona (2002), Basaldúa (2007) y Trejo (2007) que son: fiscalización, recaudación y facilitación del comercio exterior, no obstante que en este estudio el enfoque se dará sobre el efecto en la función esencial de la aduana de facilitación comercial.

TABLA 1. Síntesis de LAS FUNCIONES QUE COMPONEN LA GESTIÓN ADUANERA

\begin{tabular}{|c|c|c|c|}
\hline Autores & Funciones de la aduana & Objetivo que se busca & $\begin{array}{c}\text { Síntesis de las funciones } \\
\text { que componen la gestión } \\
\text { aduanera }\end{array}$ \\
\hline \multirow[t]{4}{*}{ Barahona (2002) } & Recaudación fiscal & \multirow{14}{*}{$\begin{array}{l}\text { Equilibrio entre facilitación comercial, } \\
\text { fiscalización y su correspondiente } \\
\text { control }\end{array}$} & \multirow{14}{*}{$\begin{array}{l}\text { Fiscalización } \\
\text { Recaudación } \\
\text { Facilitación comercial }\end{array}$} \\
\hline & $\begin{array}{l}\text { Control del tráfico internacional de } \\
\text { mercancías }\end{array}$ & & \\
\hline & Facilitación del comercio & & \\
\hline & Generación de información & & \\
\hline \multirow[t]{2}{*}{ Basaldúa (2007) } & Control del Estado & & \\
\hline & Facilitación comercial & & \\
\hline \multirow[t]{8}{*}{ Trejo (2007) } & Facilitación comercial & & \\
\hline & Recaudación & & \\
\hline & Fiscalización & & \\
\hline & De verificación & & \\
\hline & De prevención & & \\
\hline & De estadística & & \\
\hline & De respeto a los derechos humanos & & \\
\hline & De seguridad & & \\
\hline
\end{tabular}

Fuente: Reyes (2018).

Finalmente, se han ubicado bastantes análisis o estudios previos relacionados sobre los efectos de la modernización aduanera, sin embargo, de acuerdo a los objetivos de esta indagación resulta preponderante conocer desde que dimensión de la modernización se generan los impactos en la recaudación, además de los beneficios directos e indirectos que se tienen como resultado. 


\section{La modernización de la aduana en México y la facilitación comercial}

En concordancia con las propuestas de la OMA contenidas en el convenio de Kyoto y el Marco SAFE, el gobierno federal de México y la Administración General de Aduanas (AGA) implementaron el programa aduana modelo (PAM) y el plan de modernización de las aduanas 2007-2012 (PMA), como ejes de las acciones de la modernización aduanera en México.

Tanto el PAM como el PMA 2007-2012, fueron en su época los proyectos más relevantes e innovadores tratándose de la modernización en las aduanas, el primero de los mencionados, inserto las mejores prácticas del SAT relacionadas con el análisis de procesos y el proceso de reclutamiento de personal, (Servicio de Administración Tributaria, 2015b), prácticas que si bien es cierto se limitaron en un inicio únicamente a las aduanas de Veracruz, Manzanillo, Ciudad Reynosa y Nuevo Laredo, debido a que según datos disponibles para el 2006, en su conjunto participaban con un $46 \%$ de la recaudación del comercio exterior y despachaban el $25 \%$ de las operaciones de comercio exterior del país, pero en la actualidad son políticas generalizadas en las 49 aduanas mexicanas, por otro lado el PMA 20072012 se ajustó a los objetivos del SAT, tratando de ir acorde a la generalidad de las iniciativas, proyectos y planes impulsados por este último, la ejecución del PMA se dio a partir de una planificación estratégica que identifico las áreas prioritarias de atención de la institución con el objeto de alcanzar el fortalecimiento y ordenamiento de sus competencias e incrementar la capacidad de su operación, su relación con los intervinientes externos (importadores, exportadores, agentes y apoderados aduanales, principalmente) y fortalecer el quehacer del personal aduanero, en este ultimo de manera particular se consideró a la aduana de Lázaro Cárdenas.

Por último, las apuestas más recientes para la modernización de las aduanas mexicanas se ubican el Módulo de Administración Tributaria del Comercio Exterior (MAT-CE) y el Proyecto de Integración Tecnológica Aduanera (PITA), el primero dado a conocer el 5 de agosto de 2013 por el SAT, con el objetivo de actualizar los sistemas informáticos del comercio exterior con el uso de tecnologías que proporcionen simplicidad en la operación, integridad y seguridad en la información derivadas de las operaciones de comercio exterior, en búsqueda de proporcionar al contribuyente y a la institución un mejor servicio, destacándose por incorporar a los procedimientos de comercio exterior los sistemas informáticos coincidentes al módulo (Servicio de Administración Tributaria, 2016c). En lo que respecta a PITA, este fue dado a conocer el 29 de mayo de 2015, con la finalidad de optimización, vigilancia y la respuesta oportuna de las aduanas de México, lo que incluye la automatización del despacho de mercancías, reforzamiento en el control de vehículos de pasajeros en cruces fronterizos y la eficiencia de la inspección de aduanas y áreas del SAT a través de video vigilancia (Servicio de Administración Tributaria, 2015c, 2016d)

Derivado de todos los proyectos referidos, se han tenido como resultados mejoras en materia de infraestructura, equipamiento y tecnología, procesos internos, procesos de reclutamiento y plantilla de personal y mejora en la relación y percepción de los usuarios respecto de la institución, lo antes descrito tal y como lo afirma (Reyes, 2006, pp. 32-33) fundamental para el correcto funcionamiento de la administración pública aduanera.

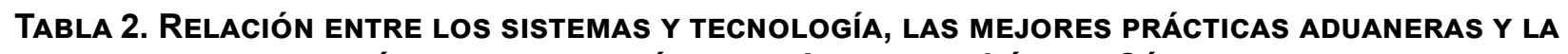
FUNCIÓN DE RECAUdACIÓN DE LA AdUANA DE LÁZARO CÁRDENAS.

\begin{tabular}{|l|l|}
\hline \multirow{2}{*}{$\begin{array}{c}\text { Prácticas } \\
\text { aduaneras }\end{array}$} & \multicolumn{1}{c|}{ Función } \\
\cline { 2 - 3 } $\begin{array}{l}\text { Función } \\
\text { pública }\end{array}$ & $\begin{array}{l}\text { Recaudación } \\
\text { Tratados internacionales en materia comercial para el incrementa la recaudación. } \\
\text { Ordenamientos administrativos a favor de importadores y exportadores para trámites de despacho de mercancías que reduce } \\
\text { los costos de importación y exportación. }\end{array}$ \\
\hline $\begin{array}{l}\text { Cambios } \\
\text { tecnológicos }\end{array}$ & $\begin{array}{l}\text { Sistemas y Mecanismos electrónicos (SAAI, SEA, VUCE, MAT-CE). } \\
\text { Programa de Integración Tecnológica Aduanera (PITA) }\end{array}$ \\
\hline Infraestructura & $\begin{array}{l}\text { Infraestructura informática. } \\
\text { Infraestructura tecnológica. }\end{array}$ \\
\hline
\end{tabular}

Fuente: Reyes (2018). 
La legislación aduanera, desde 1988 y hasta la reforma de 2018, ha contemplado el uso de la tecnología, para incrementar la eficiencia en las funciones de la aduana, particularmente, la facilitación comercial de la aduana en México. Los sistemas y tecnología usados en la Aduana de Lázaro Cárdenas para el desarrollo de sus funciones son: Sistema Automatizado Aduanero Integral (SAAI), Ventanilla Única de Comercio Exterior (VUCE), Sistema Electrónico Aduanero (SEA), mecanismo de selección automatizada, MAT-CE y los mecanismos no intrusivos de revisión. La relación entre estos, las mejores prácticas en la Aduana de Lázaro Cárdenas y su función de facilitación del comercio exterior, se presenta en la tabla 2 que evidencia la posición que tienen las mejores prácticas, la variedad y progresos en cuanto a mecanismos y sistemas en permanente actualización que surgen con la finalidad que esta aduana, contribuya eficiente y productivamente en la cadena logística nacional e internacional (Reyes, 2018).

Las reformas legales, sistemas y tecnología e infraestructura usada por la aduana para la ejecución de su función de facilitación comercial, ha rendido buenos frutos, teniendo como ejemplo lo acaecido durante el período 2007 al 2018, en el que México mejora su Índice de Desempeño Logístico o LPI, que si bien es cierto, tuvo una ligera baja en su puntaje de 3.11 en el 2016 a 3.05 en el 2018, también es cierto que en el ranking del 2018 avanzo tres posiciones colocándose en el lugar 51 respecto del 2016, que se ubicó en el lugar 54 (World Bank, 2018), haciendo énfasis en los indicadores de puntualidad de entrega de los embarques y organización de envíos competitivos o embarques internacionales y la competitividad y calidad de los servicios logísticos, pese a que existen áreas de oportunidad en lo que respecta a eficiencia en el despacho aduanero y la infraestructura, colocándose como el tercer mejor país de Latinoamérica y el caribe detrás de Panamá (lugar 38) y Chile (lugar 34) (World Bank, 2018).

\section{Método de investigación}

El método dialéctico, en el que se basa esencialmente esta investigación, debido a que este método permite el análisis y la síntesis en la fundamentación teórica del problema, así como analizar el panorama de las aduanas en México, particularmente en la de Lázaro Cárdenas, Michoacán. La aplicación de este método según lo expuesto por Reyes (2018) permite además identificar lagunas entre el progreso de la modernización aduanera en México y la facilitación del comercio exterior como función esencial que integra la gestión pública de la aduana y el menester de determinar en qué medida se produce un efecto de las mejores prácticas aduaneras como producto de la modernización de la aduana en la facilitación del comercio exterior de la administración pública aduanera y que el reconocimiento de estas brechas permita propiciar el incremento de la eficiencia de su gestión. El método inducción-deducción se usó para la construcción de las hipótesis que se presentan el cuadro 1.

De acuerdo a Reyes (2018) y Coyle et al. (2014) la técnica cuantitativa que coadyuva a alcanzar la finalidad de esta investigación es el análisis factorial confirmatorio que utiliza los Modelos de Ecuaciones Estructurales, mejor identificado en la literatura como SEM acrónimo en idioma inglés, la elección de estos modelos se justifica en virtud de los argumentos expuestos por Hair et al. (1999, pp. 612, 617, 618, 620).

Según Hair et al. (1999) para el desarrollo de esta técnica deben seguirse siete pasos indispensables, que serán considerados en esta investigación: el desarrollo de un modelo teóricamente fundado, elaborar un diagrama de secuencia de relaciones causales, transformar el diagrama de secuencia en un conjunto de modelos y relaciones estructurales, seleccionar la matriz de entrada y estimar el modelo propuesto, la evaluación de la identificación del modelo, valoración de los criterios de calidad de ajuste, interpretación y posibles cambios al modelo original propuesto.

A partir del fundamento teórico examinado y los objetivos planteados en esta investigación, se empleara el modelo medible de modernización aduanera con variables latentes y variables observadas obtenidas a través de una encuesta propuesto por Reyes (2018).

Con base en Reyes (2018), las variables dependientes son: fiscalización sobre las operaciones de comercio exterior (FA), recaudación tributaria del comercio exterior (RCEA), facilitación del comercio exterior de la aduana (FCEA), siendo esta ultima la de interés para los fines de este estudios; afectadas por la función pública (FP), cambios tecnológicos (CTN) e infraestructura (INF) como variables explicativas que conforman el constructo modernización aduanera.

En el cuadro 1 se resumen los resultados de los tres primeros pasos, comprendiendo las cuatro hipótesis que se formulan a partir del constructo teórico considerado. 


\section{CuAdRo 1. Resultados de los Pasos UnO Y dos (VARIABLES, HIPÓTESIS Y DIAGRAMA DE SECUENCIA DE RELACIONES CAUSALES)}

\begin{tabular}{|c|c|c|}
\hline Variables & Hipótesis & $\begin{array}{c}\text { Segundo paso: diagrama de secuencia } \\
\text { de relaciones causales }\end{array}$ \\
\hline \multicolumn{3}{|c|}{ Modelo estructural de modernización aduanera con variables latentes } \\
\hline $\begin{array}{l}\text { Variables explicativas } \\
\text { (constructos) y simultá- } \\
\text { neamente intervinientes: } \\
\text { función pública (FP), } \\
\text { cambios tecnológicos } \\
\text { (CTN), Infraestructura } \\
\text { (INF). } \\
\text { Variables dependientes: } \\
\text { Fiscalización de las opera- } \\
\text { ciones de comercio exte- } \\
\text { rior (FA), recaudación tri- } \\
\text { butaria del comercio exte- } \\
\text { rior (RCEA), facilitación co- } \\
\text { mercial de la aduana } \\
\text { (FCEA). }\end{array}$ & $\begin{array}{l}\text { H1: La función pública, los cambios tecno- } \\
\text { lógicos e infraestructura son factores } \\
\text { con interacción que tienen un efecto po- } \\
\text { sitivo y significativo sobre las funciones } \\
\text { esenciales de la Aduana de Lázaro Cár- } \\
\text { denas, Michoacán, México. } \\
\text { H2. La función pública es un factor que in- } \\
\text { terviene de manera positiva y significa- } \\
\text { tiva sobre la recaudación de la Aduana } \\
\text { de Lázaro Cárdenas, Michoacán, Mé- } \\
\text { xico. } \\
\text { H3: Los cambios tecnológicos son un fac- } \\
\text { tor que interviene de manera positiva y } \\
\text { significativa sobre la recaudación de la } \\
\text { Aduana de Lázaro Cárdenas, Michoa- } \\
\text { cán, México. } \\
\text { H4: La infraestructura es un factor que in- } \\
\text { terviene de manera positiva y significa- } \\
\text { tiva sobre la recaudación de la Aduana } \\
\text { de Lázaro Cárdenas, Michoacán, Mé- } \\
\text { xico. }\end{array}$ & \\
\hline
\end{tabular}

Fuente: Elaboración propia a partir de Reyes (2018).

Paso 4: Elección de la matriz de entrada y estimación del modelo propuesto

Reyes (2018) afirma que en SEM se ha empleado la matriz de varianza-covarianza o de correlación. Para este estudio se hace empleo del programa AMOS (Analysis of Moment Structures) (Arbuckle, 1994) en su versión 24.0 agregado al SPSS versión 21, la entrada de la información es a partir de datos codificados en una hoja de cálculo de SPSS, mismo que cuando es utilizado para tal efecto, crea matrices de varianza y covarianza y de correlación del total de los indicadores usados en el modelo.

Como datos para el modelo, se tomó una muestra de 254 observaciones a partir de la aplicación de un cuestionario a individuos que efectúan diferentes trámites relacionados con importaciones y exportaciones ante la Aduana de Lázaro Cárdenas. Para determinar la muestra se consideró el criterio de Palacios y Vargas (2012, p. 210) citados en Reyes (2018), que sugieren hacer uso de las tablas mostradas por Cohen (1988) y Green (1991)100 subjects.

En el cuestionario se les pidió a los individuos que valoren en qué magnitud la función pública, los cambios tecnológicos y la infraestructura son factores que influyen positiva y significativamente en la fiscalización, recaudación y facilitación comercial de la Aduana de Lázaro Cárdenas, Michoacán, México. Se usó una escala tipo Likert de 1 a 5 (1 "importancia concedida muy baja" a 5 "importancia concedida muy alta") adecuada partiendo del trabajo de León (2012).

Teniendo los cuestionarios contestados, Hair et al. (1999, pp. 63-65) sugiere valorar las características distribucionales de las variables individuales que se incluyen en los modelos. Una hipótesis fundamental del análisis multivariante es la normalidad de los datos, por eso es que el autor de cita aconseja el análisis de curtosis, test estadístico de normalidad como el valor estadístico $z$, entre otros. Afirmando que si el valor estadístico de $z$ excede $+/-2,58$ se señala que es posible rechazar el supuesto de normalidad de la distribución a un nivel de probabilidad de 0,01.

Sin embargo, Hair et al. (1999, p. 633) plantean soluciones a nivel de técnicas de estimación de los modelos estructurales y de medida. Por lo que se sugieren técnicas de estimación como mínimos cuadrados generalizados (GLS) y asintóticamente libre de distribución (AGL). Al final para esta investigación se usó la técnica GLS, de acuerdo a lo establecido en Díez (1992), ya que esta es apropiada para el análisis de variables dicotómicas u ordinales como las empleadas en esta investigación.

Paso 5: Valoración de la identificación del modelo En el desarrollo de la estimación se pueden presentar re- 
sultados incongruentes que dejan en evidencia dificultades en la identificación del modelo estructural, que son los denominados estimados ofensivos, reconociéndose como las tres razones más comunes por las que se presentan las siguientes: estimados negativos de varianza del término de error, coeficientes beta estandarizados con valores muy acercados o por encima de 1, excesivos errores estándar de estimación.

Siendo el caso que, que no se observaron en ninguna situación varianzas de error negativas; las varianzas explicadas por las variables observables se encuentran dentro del rango admitido, tal y como las estimaciones de los parámetros estandarizados con su respectivo error estándar aproximado. No se visualizaron coeficientes cercanos a 10 por encima de este.

Al mismo tiempo se analizaron los criterios de ajuste del modelo mediante los grados de libertad, que resultan de diferencia entre el número de correlaciones o covarianzas y el número efectivo de coeficientes en el modelo que se propone (Hair et al., 1999, p. 635). El modelo tiene 24 grados de libertad. Ahora bien el vínculo entre chi cuadrado y los grados de libertad o chi-cuadrado normada $\left(\mathrm{x}^{2} / \mathrm{gl}\right)$ tiene un valor de 2.7 lo que se encuentra en el rango admitido tomando en consideración las sugerencias que van desde tan alto como 5.0 (Wheaton, Muthen, Alwin, \& Summers, 1977) y tan bajo como 2.0, citado en Hooper, Coughlan, \& Mullen, 2008).

Paso 6: Evaluación de la calidad del ajuste del modelo A partir de Palacios y Vargas (2012, p. 203), la finalidad de SEM es producir una matriz de covarianza muestral con los datos y compararla con la matriz de covarianzas poblacional del modelo teórico. Si existe buen ajuste, queda de manifiesto que existe evidencia empírica a favor del modelo teórico. Los índices de ajuste más utilizados y que se consideran básicos son: el índice comparativo de ajuste (Comparative Fit Index, o (FI) y la aproximación de la raíz del error cuadrático Medio (Root Mean Square Error Approximation, o RMSEA).

En la práctica, Browne \& Cudeck (1993, referidos en Palacios y Vargas, 2012), proponen que un RMSEA cercano a 0 significa un ajuste excelente del modelo, un valor entre 0.05 y 0.08 , un ajuste satisfactorio y un valor mayor 0.09 , un mal ajuste. Para CFI, valores aproximados a 1 señalan un buen ajuste y menores a 0.85 , mal ajuste.

Para este modelo el valor de Root Mean Square Residual $(R M R)=0.03$ (es adecuado debido a que mientras más se acerca a o mejor ajusta el modelo), Goodness-of-Fit Index $(\mathrm{GFI})=0.94$ (este índice de bondad de ajuste cuando se acerca a 1 tiene un ajuste perfecto). El RMSEA $=0.08 \mathrm{y}$ cuenta con un nivel de significación ( $P$ value) de 0.01. Los resultados antes mostrados confirman un ajuste satisfactorio, aceptándose el modelo propuesto.

Paso 7: Interpretación y potenciales adecuaciones al modelo original propuesto

A través del uso de las soluciones estandarizadas que proporciono el modelo en relación a los coeficientes que se arrojaron, es posible interpretar las relaciones entre la modernización aduanera vista desde los tres componentes y las funciones esenciales de la Aduana de Lázaro Cárdenas (Reyes, 2018) y se puede afirmar que hay un efecto positivo y significativo entre los constructos y las funciones de la aduana (todos los coeficientes estimados presentan valores superiores a 0,64).

\section{Resultados}

El primer resultado que se observa es que el efecto directo de los cambios tecnológicos es significativamente superior en la infraestructura (0.58) que en la función pública (0.35). Esta diferencia se puede explicar por los importantes cambios tecnológicos que se han efectuado en esta aduana y otras del país durante el período 2003-2016, aunque la aduana de Lázaro Cárdenas no fue considerada en el PAM, sin embargo, si lo fue en el PMA, situación que al igual que en otras aduanas vino a mejorar de manera muy ligera los procedimientos de fiscalización, siendo el principal foco de atención la necesidad de mejora en materia de infraestructura.

No obstante, el efecto directo de la infraestructura respecto de la recaudación de la aduana es 0.85 , siendo este el coeficiente estimado más alto y poderoso presentado, lo que se puede explicar por la inversión que se ha efectuado por parte de las empresas prestadoras de servicios portuarios incluyendo a la administración portuaria de Lázaro Cárdenas, aunque no propiamente se trata de infraestructura que le corresponda o administre la aduana de Lázaro Cárdenas, es evidente que los esfuerzos que desde el sector privado se están realizando en este rubro, han venido a impactar positivamente la gestión en la Aduana de Lázaro Cárdenas, tecnificando sus operaciones, beneficiando el flujo y el desaduanamiento y luego entonces al incrementarse el número de operaciones realizadas y tramitadas desde los lugares donde se encuentran depositadas las mercancías y hasta su desaduanamiento final, esto genera la posibilidad de recaudar más y mejor. 
En lo atinente a las reformas con la finalidad de mejorar los procesos, tramites y ejercicio de las facultades aduanera por la aduana de Lázaro Cárdenas, es decir, la función pública, el coeficiente resultante de este respecto de la recaudación es el más bajo de todos los resultados 0.66 , no obstante, al ser mayor que 0.5 indica grandes efectos. Lo que es un claro síntoma de los esfuerzos realizados por el gobierno federal de México por mejorar los procesos en donde, entre otras cosas, intervienen los funcionarios públicos, eligiendo a los mejores perfiles, capacitándoles e imprimiéndoles identidad por la institución.

Por último, las reformas en materia de cambios tecnológicos han tienen un efecto significativo y positivo sobre la recaudación con un coeficiente de 0.68 , lo que se explica debido a que, si bien es cierto la Aduana de Lázaro Cárdenas ha sido considerada dentro de aquellas en donde se ejecutarán los programas en materia tecnológica para mejorar la calidad de los servicios, aumentar la velocidad del flujo de las mercancías y hacer más eficiente la detección de ilícitos, estos aún están en procesos de implementación, lo que no permite que se perciban aun con mayor fuerza los efectos positivos de ellos, sin embargo, las tendencias recaudatorias en esta aduana, de acuerdo a los indicadores volumétricos de esta aduana, arrojan evidencia contundente de que tecnologías como VUCE, SEA y MATCE están mejorando la función de recaudación en esta aduana.

\section{Conclusiones}

En la actualidad, la función de la administración pública aduanera, busca un equilibrio entre las funciones de control y de facilitación del comercio exterior, lo cual de forma paulatina se ha ido alcanzado por las iniciativas gubernamentales que han puesto en la mira la necesidad de la modernización aduanera, reconocida en este trabajo por los cambios a partir de diversas reformas en materia aduanera en la función pública, cambios tecnológicos e infraestructura como resultado de las mejores prácticas en materia aduanera impulsadas a nivel mundial.

El PAM y el PMA 2007-2012, además de las iniciativas PITA y la modernización de SAAI mediante MATCE aplicados en la mayoría de las aduanas en México, se han convertido en la mejor forma de propiciar la simplificación, automatización y facilitación en las operaciones transfronterizas y la mejora en el control que ejerce la administración aduanera.

Al analizar las mejores prácticas derivadas de la modernización de la Aduana de Lázaro Cárdenas y su efecto en las funciones de su gestión aduanera a través de SEM se corrobora que:

a) La alta necesidad de la tecnificación, automatización y la inserción de los medios digitales en las operaciones de comercio exterior, se evidencia en la mejora de la recaudación donde interviene la aduana.

b) Se reconoce como una necesidad imperativa la inversión en materia de infraestructura para hacer más ágil y sencilla la movilización de mercancías, lo que permitirá el paso de altos volúmenes y que incentivará al incremento en la recaudación.

c) Respecto a los cambios en la función pública aduanera, sigue existiendo una importante coyuntura que atender, ya que aún sigue persistiendo la percepción que involucramiento de la mano del hombre, pese a la mejores de procesos y directrices institucionales, sigue siendo un factor que es considerado como un obstáculo para el flujo del comercio exterior.

Finalmente, es evidente que los esfuerzos por mejorar la recaudación de la que se encarga la Aduana de Lázaro Cárdenas, reflejan un impacto positivo proveniente de los cambios a propósito de la modernización aduanera, proviniendo con mayor fuerza de la infraestructura y los cambios tecnológicos.

\section{Referencias}

Alcantar, C., Daza, M., y Velásquez, I. (2019). La modernización y actualización del catastro en México: una alternativa para mejorar sus procesos. Revista Inclusiones Vol: 6 núm. Especial: 128-141. Recuperado de: http://www. archivosrevistainclusiones.com/gallery/8\%20vol\%20 6\%20num\%202\%202019espabriljunio19inc.pdf

Administración General de Aduanas. (2007). Plan de modernización de las aduanas 2007-2012. Sectaria de Hacienda y Crédito Publico.

Administracion portuaria integral de Lázaro Cárdenas. (2019). Acerca del Puerto [Publica]. puertolazarocardenas.com.mx. https://www.puertolazarocardenas.com. $\mathrm{mx} / \mathrm{plc25/acerca-del-puerto}$

Arbuckle, J. L. (1994). Computer announcement amos: Analysis of moment structures. Psychometrika, 59(1), 135-137.

Ávila, D. (2014). El uso de las TICs en el entorno de la nueva gestión pública mexicana. Andamios, 11(24), 263-288.

Barahona, J. (2002). La gestión aduanera de Costa Rica. En El Sistema Tributario Costarricense. Contribuciones al debate nacional (Segunda, p. 60). Publicaciones, CGR. 
Basaldúa, R. (2007). La Aduana: Concepto y funciones esenciales y contingentes. Revista de estudios aduaneros, 18(1), 37-54.

Bozeman, B. (2015). Causas, efectos y eficacia de la burocratización en las administraciones públicas nacionales: Desarrollando reformas sensibles a culturas políticas singulares. Revista del CLAD Reforma y Democracia, 63, 5-32.

Bozzi, S. O. (2014). Evaluación de la Gestion Pública: Conceptos y aplicaciones em el caso latinoamericano. Brasília. Revista do Serviço Público, ano, 52. http://seer.enap. gov.br/index.php/RSP/article/view/299

Cohen, J. (1988). Statistical Power Analysis for the Behavioral Sciences (Second). Lawrence Erlbaum Associates.

Comisión Económica para América Latina y el Caribe. (2019, abril 2). Informe de la actividad portuaria de América Latina y el Caribe 2018 [Text]. CEPAL. https://www.cepal. org/es/notas/informe-la-actividad-portuaria-america-latina-caribe-2018

Coyle, T., Cruthirds, K., Naranjo, S., \& Nobel, K. (2014). Analysis of current customs practices in the United States and a proposed model for world class Customs. World Customs Journal, 8(1), 71-86. http://worldcustomsjournal.org/Archives/Volume $\% 208 \% 2$ C $\% 20$ Number\%201\%20 (Mar\%202014)/06\%20Tosevska-Trpcevska.pdf.

Culebro, J. (2008). Atomización del Estado y nuevas formas de control: La introducción de los convenios de desempeño en organizaciones públicas. Gestión y política pública, 17(1), 35-70.

Díez, J. (1992). Métodos de análisis causal. Centro de Investigaciones Sociologicas. http://www.sidalc.net/cgi-bin/ wxis.exe/?IsisScript=AGRIUAN.xis\&method=post\&formato $=2 \&$ cantidad $=1 \&$ expresion $=\mathrm{mfn}=001589$

Doimeadiós, Y., \& Rodríguez, E. (2016). Un análisis comparado de eficiencia y eficacia en el sector público en Cuba. Revista Economía y Desarrollo, 155(2). http://www. econdesarrollo.uh.cu/index.php/EDD/article/view/47

García, I. (2007). La nueva gestión pública: Evolución y tendencias. Presupuesto y Gasto público, 47, 37-64.

Green, S. (1991). How Many Subjects Does It Take To Do A Regression Analysis. Multivariate Behavioral Research, 26(3), 499-510. https://doi.org/10.1207/s15327906mbr2603_7

Gwardzińska, E. (2012). The standardisation of customs services in the European Union. World Customs Journal, 6(1), 93-100. http://worldcustomsjournal.org/Archives/Volume $\% 206 \% 2$ C 20 Number\%201\%20(Mar\%20 2012)/07\%20Gwardzinska.pdf.

Hair, J., Anderson, R., Tatham, R., \& Black, W. C. (1999). Aná- lisis multivariante (Quinta). Prentice Hall Iberia. http:// www.sidalc.net/cgi-bin/wxis.exe/?IsisScript=AGRISUM. $\mathrm{xis} \&$ method $=$ post\&formato $=2 \&$ cantidad $=1 \&$ expresion $=\mathrm{mfn}=000231$

Hooper, D., Coughlan, J., \& Mullen, M. (2008). Structural equation modelling: Guidelines for determining model fit. Articles, 2.

Horta, R. (2000). Revisión del Convenio de Kyoto (Primera). Centro de Investigaciones Aduanera y de Comercio Internacional, A. C.

León, F. (2012). Organizational citizenship behaviors among public employees. A structural equation modeling approach (Primera). Editorial Universitaria, Universidad de Guadalajara. https://books.google.com. $\mathrm{mx} /$ books?hl=es\&lr=\&id=WWH_AAAAQBAJ\&oi=fnd\&pg=PT3\&dq=Organizational+citicenship+behaviors+among+public+employees+a+structural+equation+modeling+approach\&ots=u6lgV6qG2h\&sig=pojHopzAhXGiU7kdRj44WxcuiyU

Morales, M. (2014). Nueva Gestión Pública en Chile. Revista de Ciencia Política, 34(2), 417-438.

Moyada, F. (2011). Gobernanza y calidad en la gestión pública. Estudios Gerenciales, 27(120), 205-223.

Convenio Internacional para la Simplificación y Armonización de los Regímenes Aduaneros, (1974). http:// www.wcoomd.org/en/topics/facilitation/instrument-and-tools/conventions

Organización Mundial de Aduanas. (2012). Marco Normativo SAFE de la OMA. Organización Mundial de aduanas; http://www.wcoomd.org/en/topics/facilitation/instrument-and-tools/tools/ /media/C6CDF626AFB348FCA2AC59B796B79833.ashx. http://www.wcoomd.org/ en/topics/facilitation/instrument-and-tools/tools/

Organización mundial de comercio. (2013, diciembre). Conferencias Ministeriales-Novena Conferencia Ministerial de la OMC. [Publica]. World Trade Organization. https:// www.wto.org/spanish/thewto_s/minist_s/mc9_s/desci36_s.htm

Organización mundial del comercio. (s. f.). OMC | La facilitación del comercio. Organización mundial del comercio. Recuperado 7 de febrero de 2017, de https://www.wto. org/spanish/tratop_s/tradfa_s/tradfa_s.htm

Palacios, J., \& Vargas, D. (2012). Medición efectiva de la calidad, innovaciones en México. Trillas.

Ponce de León, R. (2011). La administración pública en México (Primera). Editorial Porrúa S. A de C. V.

Ramiréz, M. (2013). Transformaciones del Estado en el gobierno local: La nueva gestión pública en Medellín. Reflexión Política, 14(28). http://revistas.unab.edu.co/in- 
dex.php?journal=reflexion\&page=article\&op=viewArticle\&path\%5B\%5D=1681

Reyes, E. (2006). Facilitación aduanera y comercial para una mejor logística (Primera). Bufete Internacional de Intercambio, S. A. de C. V.

Reyes, O. (2018). La fiscalización, recaudación y facilitación del comercio exterior de la aduana a partir de su modernización: El caso de la Aduana de Manzanillo, Colima, México [Doctorado]. De Guadalajara.

Reyes Real, O. B., Palos Sosa, M. E., \& de Dios Martínez, A. (2018). La fiscalización, recaudación y facilitación del comercio exterior de la aduana a partir de su modernización: Un estudio de caso. Retos de la Dirección, 12(1), 186-211.

Ruiz, J. S. (2012). Calidad en la gestión pública: Del azar a la necesidad. Revista del CLAD Reforma y democracia, 54, 63-94.

Sanchéz, J. (2011, marzo 21). Liderazgo y Empresa. http:// www.liderazgoyempresa.com/termino/freerider

Servicio de Administración Tributaria. (2006). Libro blanco 01. Aduana Modelo. ftp://ftp2.sat.gob.mx/asistencia_ftp/publicaciones/transparencia/Libros_Blancos/ archivos_8956/lb1_1.pdf. ftp://ftp2.sat.gob.mx/asistencia_ftp/publicaciones/transparencia/Libros_Blancos/

Servicio de Administración Tributaria. (2015a). Oficio de folio numero 0610100149915.

Servicio de Administración Tributaria. (2015b). Oficio de folio numero 0610100150015.

Servicio de Administración Tributaria. (2015c). Proyecto de Integración tecnologica aduanera (PITA). Anexo Técnico. Sectaria de Hacienda y Crédito Publico.

Servicio de Administración Tributaria. (2016a). Oficio de folio numero 0610100045516.
Servicio de Administración Tributaria. (2016b). Oficio de folio numero 0610100045616.

Servicio de Administración Tributaria. (2016c). Oficio de folio numero 0610100169416.

Servicio de Administración Tributaria. (2016d). Oficio de folio numero 0610100169516.

Shujie, Z., \& Shilu, Z. (2009). The implication of customs modernization on export competitiveness in China. En Chapter (Vol. 5, pp. 121-131).

Tabachnick, B. G., \& Fidell, L. S. (2007). Experimental designs using ANOVA. Thomson/Brooks/Cole.

Trejo, P. (2007). La facilitación en el comercio mundial y su impacto en la legislación aduanera mexicana: Propuesta de adhesión a la Versión Revisada del Convenio de Kyoto [Doctorado]. Universidad Nacional Autónoma de MéxiCO.

Trejo, P. (2008). Aduanas, régimen jurídico y facilitación (Primera). Porrúa.

Wheaton, B., Muthen, B., Alwin, D. F., \& Summers, G. F. (1977). Assessing reliability and stability in panel models. Sociological methodology, 8, 84-136.

World Bank. (2016). The World Bank. International LPI Global Ranking. https://lpi.worldbank.org/international/global

World Bank. (2018). Índice de desempeño logístico. https:// Ipi.worldbank.org/international/aggregated-ranking

World Trade Organization. (1998). Trade facilitation-Overview of trade facilitation work until mid-1998. World Trade Organization. https://www.wto.org/english/tratop_e/tradfa_e/tradfa_overview1998_e.htm

Zamora, A. I., \& Navarro, J. C. L. (2014). Eficiencia de la administración pública aduanera a través del modelo DEA. CONfines de relaciones internacionales y ciencia política, 10(20), 117-135. 